\title{
Mathematical justification of the apparition of the electromagnetic coupling
}

\author{
Somsak Orankitjaroen ${ }^{1,3^{*}}$ (D), Christian Licht ${ }^{1,2,3}$ and Thibaut Weller ${ }^{2}$
}

"Correspondence:

somsak.ora@mahidol.ac.th

'Department of Mathematics,

Faculty of Science, Mahidol

University, Bangkok, Thailand

${ }^{3}$ Centre of Excellence in

Mathematics, CHE, Bangkok,

Thailand

Full list of author information is

available at the end of the article

\begin{abstract}
By using the asymptotic analysis method as regards two-scale convergence we derive the effective behavior of a fine mixing of piezoelectric material and piezomagnetic material. It can be shown that an electromagnetic coupling arises only when each phase is connected and the interface is electrically and magnetically impermeable.
\end{abstract}

Keywords: Homogenization; Two-scale convergence; Piezoelectricity; Piezomagnetism

\section{Introduction}

Developing smart materials or multifunctional structures by using product properties of composite materials is a quite old idea but it, however, keeps getting more and more interesting for many technological applications. The concept is simple (see [1]): an effect in one of the phases of the composite implies a second effect in one of the other phases. Here we focus on the magnetoelectric coupling emerging in a composite material made of a magnetostrictive phase and a piezoelectric phase. This kind of situation is interesting because the direct coupling between electric and magnetic fields is seldom found in nature and, when it exists, it is very weak. The composite materials made of ferroelectric and ferromagnetic phases, however, may generate a considerably higher magnetoelectric response. This domain of research falls within the field of metamaterials which possess properties that cannot be found naturally. Understandably this opens the door for many different technological applications such as data storage, mechanical devices, magnetic sensors, high frequency signal treatment, etc. It is therefore of interest to propose an efficient and accurate modeling of the behavior of a composite material made of a piezoelectric phase and a piezomagnetic phase. This was done in [2] considering multilayered structures and using the asymptotic expansion method. It is worthwhile to refer to [3] and the references quoted therein to get a good overview of the problem. Here we propose a mathematically rigorous study of this situation by using asymptotic analysis method such as twoscale convergence. We consider a periodically heterogeneous composite material made of a piezoelectric phase and a piezomagnetic phase. We introduce two other parameters that refer to the connectedness of each phase and to the boundary conditions on the interface. Depending on these two parameters, we show that different models appear when the size of the period goes to zero. The situations when a full coupling among mechanical, electric 
and magnetic effects (i.e., nonvanishing elasto-magneto-electric coupling coefficients in the linearized context considered here) appears are highlighted.

\section{Setting the problem}

Let $Y_{\mathrm{e}}$ a domain of $\mathbb{R}^{3}$ included in $Y:=(0,1)^{3}$ with a Lipschitz-continuous boundary. For all real positive numbers $\varepsilon$, let $E_{\mathrm{e}}^{\varepsilon}:=\bigcup_{i \in \mathbb{Z}^{3}} \varepsilon\left(i+Y_{\mathrm{e}}\right)$, which is assumed to be connected in $\mathbb{R}^{3}$, and $E_{\mathrm{m}}^{\varepsilon}:=\mathbb{R}^{3} \backslash \bar{E}_{\mathrm{e}}^{\varepsilon}, Y_{\mathrm{m}}:=Y \backslash \bar{Y}_{\mathrm{e}}$. We will consider a body occupying a domain of $\mathbb{R}^{3}$ with a Lipschitz-continuous boundary $\partial \Omega$ made of a purely piezoelectric phase occupying $\Omega_{\mathrm{e}}^{\varepsilon}:=\Omega \cap E_{\mathrm{e}}^{\varepsilon}$ and a purely piezomagnetic phase occupying $\Omega_{\mathrm{m}}^{\varepsilon}:=\Omega \cap E_{\mathrm{m}}^{\varepsilon}$. So the magnetoelectromechanical state of the body is described by the triplet $s^{\varepsilon}:=\left(u^{\varepsilon}, \phi^{\varepsilon}, \psi^{\varepsilon}\right)$ where $u^{\varepsilon}, \phi^{\varepsilon}, \psi^{\varepsilon}$ denote the field of displacement, the electrical potential, the magnetic potential defined in $\Omega, \Omega_{\mathrm{e}}^{\varepsilon}, \Omega_{\mathrm{m}}^{\varepsilon}$, respectively. If $\sigma^{\varepsilon}, D^{\varepsilon}, B^{\varepsilon}$ denote the stress, the electric induction, the magnetic induction, respectively, one has

$$
\left(\sigma^{\varepsilon}, D^{\varepsilon}\right)=M_{\mathrm{e}}\left(e\left(u^{\varepsilon}\right), \nabla \phi^{\varepsilon}\right) \quad \text { in } \Omega_{\mathrm{e}}^{\varepsilon}, \quad\left(\sigma^{\varepsilon}, B^{\varepsilon}\right)=M_{\mathrm{m}}\left(e\left(u^{\varepsilon}\right), \nabla \psi^{\varepsilon}\right) \quad \text { in } \Omega_{\mathrm{m}}^{\varepsilon},
$$

where $e\left(u^{\varepsilon}\right)$ is the strain associated with $u^{\varepsilon}$ while $M_{\mathrm{e}}$ and $M_{\mathrm{m}}$ stand for the piezoelectric and piezomagnetic tensors, respectively, with $M_{\mathrm{e}}, M_{\mathrm{m}}$ in $\operatorname{Lin}\left(\mathbb{S}^{3}\right)$ satisfying

$$
\begin{array}{ll}
\exists \alpha>0 \text { s.t. } \quad & M_{\mathrm{e}}(e, h) \cdot(e, h) \geq \alpha|(e, h)|^{2}, \\
& M_{\mathrm{m}}(e, h) \cdot(e, h) \geq \alpha|(e, h)|^{2} \quad \forall(e, h) \in \mathbb{S}^{3} \times \mathbb{R}^{3},
\end{array}
$$

where $\operatorname{Lin}\left(\mathbb{S}^{3}\right)$ denotes the space of linear mappings on $\mathbb{S}^{3}$ the space of symmetric $3 \times 3$ matrices whose scalar product and norm are denoted by $\cdot$ and $|\cdot|$ as in $\mathbb{R}^{3}$.

We will consider various situations indexed by $p=\left(p_{1}, p_{2}\right)$ in $\{1,2\} \times\{1,2,3,4\}$. The case $p_{1}=1$ corresponds to $\Omega_{\mathrm{e}}^{\varepsilon}$ and $\Omega_{\mathrm{m}}^{\varepsilon}$ connected, $p_{1}=2$ corresponds to $\Omega_{\mathrm{e}}^{\varepsilon}$ connected but $\Omega_{\mathrm{m}}^{\varepsilon}$ disconnected. (Of course, by exchanging the words electric and magnetic the following results may be adapted if it is the magnetic phase only which is connected!) Let $\Gamma_{\mathrm{I}}^{\varepsilon}:=$ $\bar{\Omega} \cap \partial E_{\mathrm{e}}^{\varepsilon}\left(=\bar{\Omega} \cap \partial E_{\mathrm{m}}^{\varepsilon}\right)$ be the interface between the two phases, when

$p_{2}=1: \Gamma_{\mathrm{I}}^{\varepsilon}$ is assumed to be electrically and magnetically impermeable $\left(\partial_{n_{\mathrm{e}}} \phi^{\varepsilon}=\partial_{n_{\mathrm{m}}} \psi^{\varepsilon}=\right.$ $0)$ on $\Gamma_{\mathrm{I}}^{\varepsilon}$ where $n_{\mathrm{e}}$ and $n_{\mathrm{m}}=-n_{\mathrm{e}}$ denote the normal outward to $\Omega_{\mathrm{e}}^{\varepsilon}$ and $\Omega_{\mathrm{m}}^{\varepsilon}$, respectively;

$p_{2}=2$ : the electric and magnetic potentials are assumed to be constant on each connected component of $\Gamma_{\mathrm{I}}^{\varepsilon}$;

$p_{2}=3: \Gamma_{\mathrm{I}}^{\varepsilon}$ is assumed to be electrically impermeable while the magnetic potential has to be constant on each connected component of $\Gamma_{\mathrm{I}}^{\varepsilon}$;

$p_{2}=4$ : the role played by electricity and magnetism in the previous case $\left(p_{2}=3\right)$ is exchanged.

Eventually, we assume that the electric and magnetic potentials take given values $\phi_{0}$ and $\psi_{0}$ on $\Gamma_{\mathrm{e}, \text { ext }}^{\varepsilon}:=\partial \Omega \cap \bar{E}_{\mathrm{e}}^{\varepsilon}$ and $\Gamma_{\mathrm{m} \text {,ext }}^{\varepsilon}:=\partial \Omega \cap \bar{E}_{\mathrm{m}}^{\varepsilon}, \phi_{0}$ and $\psi_{0}$ being fields defined on $\bar{\Omega}$ that we assume to be constant when $p_{2} \neq 1$. In all cases, the body is clamped on $\Gamma_{\mathrm{D}} \subset \partial \Omega$ which is assumed to be of positive two-dimensional Hausdorff measure, subjected to body and surface forces on $\Gamma_{\mathrm{N}}=\partial \Omega \backslash \Gamma_{\mathrm{D}}$ of densities $f$ and $g$ and there are no electric or magnetic charges. So if we assume that $\phi_{0}$ and $\psi_{0}$ belong to $H^{1}(\Omega)$ and $(f, g)$ to 
$L^{2}\left(\Omega ; \mathbb{R}^{3}\right) \times L^{2}\left(\Gamma_{\mathrm{N}} ; \mathbb{R}^{3}\right)$, a weak formulation of the equilibrium problem for the body is

$$
\left(\mathcal{P}^{\varepsilon}\right)\left\{\begin{array}{l}
\text { Find } s^{\varepsilon}=\left(u^{\varepsilon}, \phi^{\varepsilon}, \psi^{\varepsilon}\right) \text { in }\left(0, \phi_{0}, \psi_{0}\right)+H_{\Gamma_{\mathrm{D}}}^{1}\left(\Omega ; \mathbb{R}^{3}\right) \times V_{\mathrm{e}, p_{2}}^{\varepsilon} \times V_{\mathrm{m}, p_{2}}^{\varepsilon} \text { s.t. } \\
\int_{\Omega_{\mathrm{e}}^{\varepsilon}} M_{\mathrm{e}}\left(e\left(u^{\varepsilon}\right), \nabla \phi^{\varepsilon}\right) \cdot(e(v), \nabla \phi) d x+\int_{\Omega_{\mathrm{m}}^{\varepsilon}} M_{\mathrm{m}}\left(e\left(u^{\varepsilon}\right), \nabla \psi^{\varepsilon}\right) \cdot(e(v), \nabla \psi) d x \\
\quad=\int_{\Omega} f \cdot v d x+\int_{\Gamma_{\mathrm{N}}} g \cdot v d \mathcal{H}_{2} \quad \forall(v, \phi, \psi) \in H_{\Gamma_{\mathrm{D}}}^{1}\left(\Omega ; \mathbb{R}^{3}\right) \times V_{\mathrm{e}, p_{2}}^{\varepsilon} \times V_{\mathrm{m}, p_{2}}^{\varepsilon}
\end{array}\right.
$$

with

$$
\begin{aligned}
V_{\mathrm{e}, 1}^{\varepsilon}:= & H_{\Gamma_{\mathrm{e}, \mathrm{ext}}^{\varepsilon}}^{1}\left(\Omega_{\mathrm{e}}^{\varepsilon}\right), \\
V_{\mathrm{m}, 1}^{\varepsilon}:= & \left\{\psi \in H_{\Gamma_{\mathrm{m}, \mathrm{ext}}}^{1}\left(\Omega_{\mathrm{m}}^{\varepsilon}\right)\right. \text { with vanishing average on each connected } \\
& \text { component of } \left.\Omega_{\mathrm{m}}^{\varepsilon} \text { whose boundary does not meet } \Omega\right\} ; \\
V_{\mathrm{e}, 2}^{\varepsilon}:= & H_{0}^{1}\left(\Omega_{\mathrm{e}}^{\varepsilon}\right), \\
V_{\mathrm{m}, 2}^{\varepsilon}:= & \left\{\psi \in H_{\Gamma_{\mathrm{m}, \mathrm{ext}}}^{1}\left(\Omega_{\mathrm{m}}^{\varepsilon}\right) \text { such that for all connected components } \Omega_{\mathrm{m}}^{\varepsilon, i} \text { of } \Omega_{\mathrm{m}}^{\varepsilon}\right. \\
& \text { there exists } \left.C^{\varepsilon, i}(\psi) \text { in } \mathbb{R} \text { with } \bar{\psi}:=\psi-C^{\varepsilon, i}(\psi) \text { in } H_{0}^{1}\left(\Omega_{\mathrm{m}}^{\varepsilon, i}\right)\right\} ; \\
V_{\mathrm{e}, 3}^{\varepsilon}:= & V_{\mathrm{e}, 1}^{\varepsilon}, \quad V_{\mathrm{m}, 3}^{\varepsilon}=V_{\mathrm{m}, 2}^{\varepsilon} ; \\
V_{\mathrm{e}, 4}^{\varepsilon}:= & V_{\mathrm{e}, 2}^{\varepsilon}, \quad V_{\mathrm{m}, 3}^{\varepsilon}=V_{\mathrm{m}, 1}^{\varepsilon},
\end{aligned}
$$

where for all open sets $G$ of $\mathbb{R}^{n}, H_{\gamma}^{1}\left(G ; \mathbb{R}^{n}\right)$ denotes the subspace of the Sobolev space $H^{1}\left(G ; \mathbb{R}^{n}\right)$ made of the elements with vanishing trace on $\gamma$ included in the boundary $\partial G$ of $G$.

By the Lax-Milgram lemma, $\left(\mathcal{P}^{\varepsilon}\right)$ has a unique solution $s^{\varepsilon}$. The very question is to study the asymptotic behavior when $\varepsilon$ goes to zero which will supply the effective behavior of the heterogeneous body.

\section{A convergence result}

To study the convergence when $\varepsilon$ goes to zero, we use the two-scale convergence method [4] and recall the definition.

Definition 1 A sequence of functions $v^{\varepsilon}$ in $L^{2}\left(\Omega ; \mathbb{R}^{3}\right)$ is said to two-scale converge to a limit $v^{0}$ belonging to $L^{2}\left(\Omega \times Y ; \mathbb{R}^{3}\right)$ if for any function $\theta$ in $\mathcal{D}\left(\Omega ; C_{\#}^{\infty}\left(Y ; \mathbb{R}^{3}\right)\right)$, we have

$$
\lim _{\varepsilon \rightarrow 0} \int_{\Omega} v^{\varepsilon}(x) \cdot \theta(x, x / \varepsilon) d x=\int_{\Omega \times Y} v^{0}(x, y) \cdot \theta(x, y) d x d y,
$$

where $\mathcal{D}\left(\Omega ; C_{\#}^{\infty}\left(Y ; \mathbb{R}^{3}\right)\right)$ denotes the space of infinitely smooth and compactly supported functions in $\Omega$ with values in the space $C_{\#}^{\infty}\left(Y ; \mathbb{R}^{3}\right)$ of infinitely smooth and $Y$-periodic functions.

For any element $\theta$ of $L^{2}\left(\Omega_{e}^{\varepsilon} ; \mathbb{R}^{n}\right)$ or $L^{2}\left(\Omega_{m}^{\varepsilon} ; \mathbb{R}^{n}\right)$ we denote the extension by 0 to the remaining part of $\Omega$ by $\widetilde{\theta}$. By taking $(v, \phi, \psi)=s^{\varepsilon}-\left(0, \phi_{0}, \psi_{0}\right)$ in the variational formulation of $\left(\mathcal{P}^{\varepsilon}\right)$ we deduce that $\left(\mathcal{u}^{\varepsilon}, \widetilde{\nabla \phi^{\varepsilon}}, \widetilde{\nabla \psi^{\varepsilon}}\right)$ are bounded in $H_{\Gamma_{\mathrm{D}}}^{1}\left(\Omega ; \mathbb{R}^{3}\right) \times L^{2}\left(\Omega ; \mathbb{R}^{3}\right) \times L^{2}\left(\Omega ; \mathbb{R}^{3}\right)$. The Poincaré or Poincaré-Wirtinger inequalities and the sharp estimate of [5]

$$
\begin{aligned}
& \exists C(\Omega)>0 \quad \text { s.t. } \\
& \int_{\Omega_{\mathrm{e}}^{\varepsilon}}|v|^{2} d x \leq C(\Omega) \int_{\Omega_{\mathrm{e}}^{\varepsilon}}|\nabla v|^{2} d x \quad \forall v \in H_{\Gamma_{\mathrm{e}, \mathrm{ext}}^{\varepsilon}}^{1}\left(\Omega_{\mathrm{e}}^{\varepsilon}\right),
\end{aligned}
$$




$$
\int_{\Omega_{\mathrm{m}}^{\varepsilon}}|v|^{2} d x \leq C(\Omega) \int_{\Omega_{\mathrm{m}}^{\varepsilon}}|\nabla v|^{2} d x \quad \forall v \in H_{\Gamma_{\mathrm{m}, \mathrm{ext}}^{\varepsilon}}^{1}\left(\Omega_{\mathrm{m}}^{\varepsilon}\right) \quad \text { when } \Omega_{\mathrm{m}}^{\varepsilon} \text { is connected }
$$

show that $\widetilde{\phi^{\varepsilon}}$ is bounded in $L^{2}(\Omega)$ when $p_{2}=1$ or 3 , the sequence $\left(\widetilde{\phi^{\varepsilon}-\phi_{0}}\right) / \varepsilon$ is bounded in $L^{2}(\Omega)$ when $p_{2}=2$ or $4, \widetilde{\psi^{\varepsilon}}$ is bounded in $L^{2}(\Omega)$ when $p=(1,1),(1,4),\left(\widetilde{\psi^{\varepsilon}-\psi_{0}}\right) / \varepsilon$ is bounded in $L^{2}(\Omega)$ when $p=(1,2),(1,3),(2,1),(2,4)$, the sequence $\left(\psi^{\varepsilon}-C^{\varepsilon}\left(\psi^{\varepsilon}\right)\right) / \varepsilon$ is bounded in $L^{2}(\Omega)$ when $p=(2,2)$ or $(2,3), C^{\varepsilon}\left(\psi^{\varepsilon}\right)$ being equal to $C^{\varepsilon, i}\left(\psi^{\varepsilon}\right)$ on the connected component $\Omega_{m}^{\varepsilon, i}$ of $\Omega_{\mathrm{m}}^{\varepsilon}$. Then a standard result of two-scale convergence theory implies the following.

Theorem 1 Let $\chi_{\mathrm{e}}$ and $\chi_{\mathrm{m}}$ be the characteristic functions of $Y_{\mathrm{e}}$ and $Y_{\mathrm{m}}$, then there exists a unique $\left(u^{0}, u^{1}, \phi^{0}, \phi^{1}, \psi^{0}, \psi^{1}\right)$ in $H_{\Gamma_{\mathrm{D}}}^{1}\left(\Omega ; \mathbb{R}^{3}\right) \times L^{2}\left(\Omega ; H_{\#}^{1}\left(Y_{\mathrm{m}}\right) / \mathbb{R}^{3}\right) \times\left(\phi_{0}+H_{0}^{1}(\Omega)\right) \times$ $L^{2}\left(\Omega ; H_{\#}^{1}\left(Y_{\mathrm{e}}\right) / \mathbb{R}\right) \times\left(\psi_{0}+H_{0}^{1}(\Omega)\right) \times L^{2}\left(\Omega ; H_{\#}^{1}\left(Y_{\mathrm{m}}\right) / \mathbb{R}\right)$ such that $u^{\varepsilon}$ converges weakly toward $u^{0}$ in $H_{\Gamma_{\mathrm{D}}}^{1}\left(\Omega ; \mathbb{R}^{3}\right)$ and $e\left(u^{\varepsilon}\right)$ two-scale converges toward $e\left(u^{0}\right)+e_{y}\left(u^{1}\right)$ and

$p=(1,1):\left(\widetilde{\phi^{\varepsilon}}, \widetilde{\psi^{\varepsilon}}\right)$ and $\left(\widetilde{\nabla \phi^{\varepsilon}}, \widetilde{\nabla \psi^{\varepsilon}}\right)$ two-scale converge toward $\left(\chi_{\mathrm{e}} \phi^{0}, \chi_{\mathrm{m}} \psi^{0}\right)$ and $\left(\chi_{\mathrm{e}}\left(\nabla \phi^{0}+\nabla_{y} \phi^{1}\right), \chi_{\mathrm{m}}\left(\nabla \psi^{0}+\nabla_{y} \psi^{1}\right)\right)$, respectively, with

$$
\left\{\begin{array}{c}
\left(u^{0}, u^{1}, \phi^{0}, \phi^{1}, \psi^{0}, \psi^{1}\right) \in\left(0,0, \phi_{0}, 0, \psi_{0}, 0\right)+V_{1,1} ; \\
\int_{\Omega \times Y} \chi_{\mathrm{e}}(y) M_{\mathrm{e}}\left(e\left(u^{0}\right)+e_{y}\left(u^{1}\right), \nabla \phi^{0}+\nabla_{y} \phi^{1}\right) \\
\quad \cdot\left(e\left(u^{\prime}\right)+e_{y}\left(u^{\prime 1}\right), \nabla \phi^{\prime}+\nabla_{y} \phi^{\prime 1}\right) d x d y \\
\quad+\int_{\Omega \times Y} \chi_{\mathrm{m}}(y) M_{\mathrm{m}}\left(e\left(u^{0}\right)+e_{y}\left(u^{1}\right), \nabla \psi^{0}+\nabla_{y} \psi^{1}\right) \\
\quad \cdot\left(e\left(u^{\prime}\right)+e_{y}\left(u^{\prime 1}\right), \nabla \psi^{\prime}+\nabla_{y} \psi^{\prime 1}\right) d x d y \\
=L\left(u^{\prime}\right) \quad \forall\left(u^{\prime}, u^{\prime 1}, \phi^{\prime}, \phi^{\prime 1}, \psi^{\prime}, \psi^{\prime 1}\right) \in V_{1,1},
\end{array}\right.
$$

$p=(2,1):\left(\widetilde{\phi^{\varepsilon}},\left(\widetilde{\psi^{\varepsilon}-\psi_{0}}\right) / \varepsilon\right)$ and $\left(\widetilde{\nabla \phi^{\varepsilon}}, \widetilde{\nabla \psi^{\varepsilon}}\right)$ two-scale converge toward $\left(\chi_{\mathrm{e}} \phi^{0}, \psi^{1}\right)$ and $\left(\chi_{\mathrm{e}}\left(\nabla \phi^{0}+\nabla_{y} \phi^{1}\right), \chi_{\mathrm{m}} \nabla_{y} \psi^{1}\right)$, respectively, with

$$
\left\{\begin{array}{l}
\left(u^{0}, u^{1}, \phi^{0}, \phi^{1}, \psi^{1}\right) \in\left(0,0, \phi_{0}, 0,0\right)+V_{2,1} \\
\int_{\Omega \times Y} \chi_{\mathrm{e}}(y) M_{\mathrm{e}}\left(e\left(u^{0}\right)+e_{y}\left(u^{1}\right), \nabla \phi^{0}+\nabla_{y} \phi^{1}\right) \\
\quad \cdot\left(e\left(u^{\prime}\right)+e_{y}\left(u^{\prime 1}\right), \nabla \phi^{\prime}+\nabla_{y} \phi^{\prime 1}\right) d x d y \\
\quad+\int_{\Omega \times Y} \chi_{\mathrm{m}}(y) M_{\mathrm{m}}\left(e\left(u^{0}\right)+e_{y}\left(u^{1}\right), \nabla_{y} \psi^{1}\right) \cdot\left(e\left(u^{\prime}\right)+e_{y}\left(u^{\prime 1}\right), \nabla_{y} \psi^{\prime 1}\right) d x d y \\
=L\left(u^{\prime}\right) \quad \forall\left(u^{\prime}, u^{\prime 1}, \phi^{\prime}, \phi^{\prime 1}, \psi^{\prime 1}\right) \in V_{2,1},
\end{array}\right.
$$

$p=(1,2):\left(\left(\widetilde{\phi^{\varepsilon}-\phi_{0}}\right) / \varepsilon,\left(\widetilde{\psi^{\varepsilon}-\psi_{0}}\right) / \varepsilon\right)$ and $\left(\widetilde{\nabla \phi^{\varepsilon}}, \widetilde{\nabla \psi^{\varepsilon}}\right)$ two-scale converge toward $\left(\phi^{1}, \psi^{1}\right)$ and $\left(\nabla_{y} \phi^{1}, \nabla_{y} \psi^{1}\right)$, respectively, with

$$
\left\{\begin{array}{l}
\left(u^{0}, u^{1}, \phi^{1}, \psi^{1}\right) \in V_{1,2} ; \\
\int_{\Omega \times Y} \chi_{\mathrm{e}}(y) M_{\mathrm{e}}\left(e\left(u^{0}\right)+e_{y}\left(u^{1}\right), \nabla_{y} \phi^{1}\right) \cdot\left(e\left(u^{\prime}\right)+e_{y}\left(u^{\prime 1}\right), \nabla_{y} \phi^{\prime 1}\right) d x d y \\
\quad+\int_{\Omega \times Y} \chi_{\mathrm{m}}(y) M_{\mathrm{m}}\left(e\left(u^{0}\right)+e_{y}\left(u^{1}\right), \nabla_{y} \psi^{1}\right) \cdot\left(e\left(u^{\prime}\right)+e_{y}\left(u^{\prime 1}\right), \nabla_{y} \psi^{\prime 1}\right) d x d y \\
\quad L\left(u^{\prime}\right) \quad \forall\left(u^{\prime}, u^{\prime 1}, \phi^{\prime 1}, \psi^{\prime 1}\right) \in V_{1,2},
\end{array}\right.
$$


$p=(2,2):\left(\left(\widetilde{\phi^{\varepsilon}-\phi_{0}}\right) / \varepsilon,\left(\psi^{\varepsilon} \widetilde{-C^{\varepsilon}}\left(\psi^{\varepsilon}\right)\right) / \varepsilon\right)$ and $\left(\widetilde{\nabla \phi^{\varepsilon}}, \widetilde{\nabla \psi^{\varepsilon}}\right)$ two-scale converge toward $\left(\phi^{1}, \psi^{1}\right)$ and $\left(\nabla_{y} \phi^{1}, \nabla_{y} \psi^{1}\right)$, respectively, with

$$
\left\{\begin{array}{l}
\left(u^{0}, u^{1}, \phi^{1}, \psi^{1}\right) \in V_{2,2} ; \\
\int_{\Omega \times Y} \chi_{\mathrm{e}}(y) M_{\mathrm{e}}\left(e\left(u^{0}\right)+e_{y}\left(u^{1}\right), \nabla_{y} \phi^{1}\right) \cdot\left(e\left(u^{\prime}\right)+e_{y}\left(u^{\prime}\right), \nabla_{y} \phi^{\prime 1}\right) d x d y \\
\quad+\int_{\Omega \times Y} \chi_{\mathrm{m}}(y) M_{\mathrm{m}}\left(e\left(u^{0}\right)+e_{y}\left(u^{1}\right), \nabla_{y} \psi^{1}\right) \cdot\left(e\left(u^{\prime}\right)+e_{y}\left(u^{\prime 1}\right), \nabla_{y} \psi^{\prime 1}\right) d x d y \\
\quad=L\left(u^{\prime}\right) \quad \forall\left(u^{\prime}, u^{\prime 1}, \phi^{\prime 1}, \psi^{\prime 1}\right) \in V_{2,2},
\end{array}\right.
$$

$p=(1,3):\left(\widetilde{\phi^{\varepsilon}},\left(\widetilde{\psi^{\varepsilon}-\psi_{0}}\right) / \varepsilon\right)$ and $\left(\widetilde{\nabla \phi^{\varepsilon}}, \widetilde{\nabla \psi^{\varepsilon}}\right)$ two-scale converge toward $\left(\chi_{\mathrm{e}} \phi^{0}, \psi^{1}\right)$ and $\left(\chi_{\mathrm{e}}\left(\nabla \phi^{0}+\nabla_{y} \phi^{1}\right), \nabla_{y} \psi^{1}\right)$, respectively, with

$$
\left\{\begin{array}{l}
\left(u^{0}, u^{1}, \phi^{0}, \phi^{1}, \psi^{1}\right) \in\left(0,0, \phi_{0}, 0,0\right)+V_{1,3} ; \\
\int_{\Omega \times Y} \chi_{\mathrm{e}}(y) M_{\mathrm{e}}\left(e\left(u^{0}\right)+e_{y}\left(u^{1}\right), \nabla \phi^{0}+\nabla_{y} \phi^{1}\right) \cdot\left(e\left(u^{\prime}\right)+e_{y}\left(u^{\prime}\right), \nabla \phi^{\prime}+\nabla_{y} \phi^{\prime 1}\right) d x d y \\
\quad+\int_{\Omega \times Y} \chi_{\mathrm{m}}(y) M_{\mathrm{m}}\left(e\left(u^{0}\right)+e_{y}\left(u^{1}\right), \nabla_{y} \psi^{1}\right) \cdot\left(e\left(u^{\prime}\right)+e_{y}\left(u^{\prime 1}\right), \nabla_{y} \psi^{\prime 1}\right) d x d y \\
=L\left(u^{\prime}\right) \quad \forall\left(u^{\prime}, u^{\prime 1}, \phi^{\prime}, \phi^{\prime 1}, \psi^{\prime 1}\right) \in V_{1,3},
\end{array}\right.
$$

$\left.p=(2,3):\left(\widetilde{\phi^{\varepsilon}},\left(\psi^{\varepsilon} \widetilde{-C^{\varepsilon}\left(\psi^{\varepsilon}\right.}\right)\right) / \varepsilon\right)$ and $\left(\widetilde{\nabla \phi^{\varepsilon}}, \widetilde{\nabla \psi^{\varepsilon}}\right)$ two-scale converge toward $\left(\chi_{\mathrm{e}} \phi^{0}, \psi^{1}\right)$ and $\left(\chi_{\mathrm{e}}\left(\nabla \phi^{0}+\nabla_{y} \phi^{1}\right), \nabla_{y} \psi^{1}\right)$, respectively, with

$$
\left\{\begin{array}{l}
\left(u^{0}, u^{1}, \phi^{0}, \phi^{1}, \psi^{1}\right) \in\left(0,0, \phi_{0}, 0,0\right)+V_{2,3} ; \\
\int_{\Omega \times Y} \chi_{\mathrm{e}}(y) M_{\mathrm{e}}\left(e\left(u^{0}\right)+e_{y}\left(u^{1}\right), \nabla \phi^{0}+\nabla_{y} \phi^{1}\right) \cdot\left(e\left(u^{\prime}\right)+e_{y}\left(u^{\prime 1}\right), \nabla \phi^{\prime}+\nabla_{y} \phi^{\prime 1}\right) d x d y \\
\quad+\int_{\Omega \times Y} \chi_{\mathrm{m}}(y) M_{\mathrm{m}}\left(e\left(u^{0}\right)+e_{y}\left(u^{1}\right), \nabla_{y} \psi^{1}\right) \cdot\left(e\left(u^{\prime}\right)+e_{y}\left(u^{\prime 1}\right), \nabla_{y} \psi^{\prime 1}\right) d x d y \\
=L\left(u^{\prime}\right) \quad \forall\left(u^{\prime}, u^{\prime 1}, \phi^{\prime}, \phi^{\prime 1}, \psi^{\prime 1}\right) \in V_{2,3},
\end{array}\right.
$$

$p=(1,4):\left(\left(\widetilde{\phi^{\varepsilon}-\phi_{0}}\right) / \varepsilon, \widetilde{\psi^{\varepsilon}}\right)$ and $\left(\widetilde{\nabla \phi^{\varepsilon}}, \widetilde{\nabla \psi^{\varepsilon}}\right)$ two-scale converge toward $\left(\phi^{1}, \chi_{\mathrm{m}} \psi^{0}\right)$ and $\left(\nabla_{y} \phi^{1}, \chi_{\mathrm{m}}\left(\nabla \psi^{0}+\nabla_{y} \psi^{1}\right)\right)$, respectively, with

$$
\left\{\begin{array}{l}
\left(u^{0}, u^{1}, \phi^{1}, \psi^{0}, \psi^{1}\right) \in\left(0,0,0, \psi_{0}, 0\right)+V_{1,4} \\
\int_{\Omega \times Y} \\
\quad \chi_{\mathrm{e}}(y) M_{\mathrm{e}}\left(e\left(u^{0}\right)+e_{y}\left(u^{1}\right), \nabla_{y} \phi^{1}\right) \cdot\left(e\left(u^{\prime}\right)+e_{y}\left(u^{\prime}\right), \nabla_{y} \phi^{\prime 1}\right) d x d y \\
\quad+\int_{\Omega \times Y} \chi_{\mathrm{m}}(y) M_{\mathrm{m}}\left(e\left(u^{0}\right)+e_{y}\left(u^{1}\right), \nabla \psi^{0}+\nabla_{y} \psi^{1}\right) \\
\quad \cdot\left(e\left(u^{\prime}\right)+e_{y}\left(u^{\prime 1}\right), \nabla \psi^{\prime}+\nabla_{y} \psi^{\prime 1}\right) d x d y \\
=L\left(u^{\prime}\right) \quad \forall\left(u^{\prime}, u^{\prime 1}, \phi^{\prime 1}, \psi^{\prime}, \psi^{\prime 1}\right) \in V_{1,4}
\end{array}\right.
$$

$p=(2,4):\left(\left(\widetilde{\phi^{\varepsilon}-\phi_{0}}\right) / \varepsilon,\left(\widetilde{\psi^{\varepsilon}-\psi_{0}}\right) / \varepsilon\right)$ and $\left(\widetilde{\nabla \phi^{\varepsilon}}, \widetilde{\nabla \psi^{\varepsilon}}\right)$ two-scale converge toward $\left(\phi^{1}, \psi^{1}\right)$ and $\left(\nabla_{y} \phi^{1}, \chi_{\mathrm{m}} \nabla_{y} \psi^{1}\right)$, respectively, with

$$
\left\{\begin{array}{l}
\left(u^{0}, u^{1}, \phi^{1}, \psi^{1}\right) \in V_{2,4} ; \\
\int_{\Omega \times Y} \chi_{\mathrm{e}}(y) M_{\mathrm{e}}\left(e\left(u^{0}\right)+e_{y}\left(u^{1}\right), \nabla_{y} \phi^{1}\right) \cdot\left(e\left(u^{\prime}\right)+e_{y}\left(u^{\prime}\right), \nabla_{y} \phi^{\prime 1}\right) d x d y \\
\quad+\int_{\Omega \times Y} \chi_{\mathrm{m}}(y) M_{\mathrm{m}}\left(e\left(u^{0}\right)+e_{y}\left(u^{1}\right), \nabla_{y} \psi^{1}\right) \\
\quad \cdot\left(e\left(u^{\prime}\right)+e_{y}\left(u^{\prime 1}\right), \nabla_{y} \psi^{\prime 1}\right) d x d y \\
=L\left(u^{\prime}\right) \quad \forall\left(u^{\prime}, u^{\prime 1}, \phi^{\prime 1}, \psi^{\prime 1}\right) \in V_{2,4} .
\end{array}\right.
$$


Here

$$
L\left(u^{\prime}\right):=\int_{\Omega} f \cdot u^{\prime} d x+\int_{\Gamma_{\mathrm{N}}} g \cdot u^{\prime} d x \quad \forall u^{\prime} \in H_{\Gamma_{\mathrm{D}}}^{1}\left(\Omega ; \mathbb{R}^{3}\right)
$$

and

$$
\begin{aligned}
& V_{1,1}:=K_{1} \times K_{2} \times K_{3} \times K_{4} \times K_{5} \times K_{6}, \quad V_{2,1}:=K_{1} \times K_{2} \times K_{3} \times K_{4} \times K_{6}, \\
& V_{1,2}:=K_{1} \times K_{2} \times K_{7} \times K_{8}, \quad V_{2,2}:=V_{1,2}, \\
& V_{1,3}:=K_{1} \times K_{2} \times K_{3} \times K_{4} \times K_{8}, \quad V_{2,3}:=V_{1,3}, \\
& V_{1,4}:=K_{1} \times K_{2} \times K_{7} \times K_{3} \times K_{6}, \quad V_{2,4}:=K_{1} \times K_{2} \times K_{7} \times K_{6},
\end{aligned}
$$

where

$$
\begin{aligned}
& K_{1}=H_{\Gamma_{\mathrm{D}}}^{1}\left(\Omega ; \mathbb{R}^{3}\right), \quad K_{2}=L^{2}\left(\Omega ; H_{\#}^{1}\left(Y ; \mathbb{R}^{3}\right)\right), \quad K_{3}=K_{5}=H_{0}^{1}(\Omega), \\
& K_{4}=L^{2}\left(\Omega ; H_{\#}^{1}\left(Y_{\mathrm{e}}\right) / \mathbb{R}\right), \quad K_{6}=L^{2}\left(\Omega ; H_{\#}^{1}\left(Y_{\mathrm{m}}\right) / \mathbb{R}\right), \quad K_{7}=L^{2}\left(\Omega ; H_{\#, \mathrm{~m}}^{1}(Y) / \mathbb{R}\right), \\
& K_{8}=L^{2}\left(\Omega ; H_{\#, \mathrm{e}}^{1}(Y) / \mathbb{R}\right),
\end{aligned}
$$

with

$H_{\#}^{1}\left(G ; \mathbb{R}^{n}\right)$ the completion with respect to the norm of $H^{1}\left(G ; \mathbb{R}^{n}\right)$ of the space made of the restriction to $G$ of the elements of $C_{\#}^{\infty}\left(Y ; \mathbb{R}^{n}\right)$,

$$
\begin{aligned}
& H_{\#, \mathrm{e}}^{1}(Y)=\left\{\psi \in H_{\#}^{1}(Y) \text { s.t. } \psi=0 \text { on } Y_{\mathrm{e}}\right\}, \\
& H_{\#, \mathrm{~m}}^{1}(Y)=\left\{\phi \in H_{\#}^{1}(Y) \text { s.t. } \phi=0 \text { on } Y_{\mathrm{m}}\right\} .
\end{aligned}
$$

\section{Physical interpretation}

By eliminating the microscopic variables $u^{1}, \phi^{1}, \psi^{1}$ we can characterize the nature of the effective behavior.

When $p=(1,1)$, the body has a piezoelectromagnetic behavior with an electromagnetic coupling involving the following effective piezoelectromagnetic tensor:

$$
\begin{aligned}
\mathcal{M}_{\mathrm{eff}}^{k l}:= & \int_{Y} \chi_{\mathrm{e}}(y) M_{\mathrm{e}}(y)\left[\left(E_{s}^{k}, E_{\mathrm{e}}^{k}\right)+\left(e\left(u^{k}\right), \nabla \phi^{k}\right)\right] \cdot\left[\left(E_{s}^{l}, E_{\mathrm{e}}^{l}\right)+\left(e\left(u^{l}\right), \nabla \phi^{l}\right)\right] d y \\
& +\int_{Y} \chi_{\mathrm{m}}(y) M_{\mathrm{m}}(y)\left[\left(E_{s}^{k}, E_{\mathrm{m}}^{k}\right)+\left(e\left(u^{k}\right), \nabla \psi^{k}\right)\right] \cdot\left[\left(E_{s}^{l}, E_{\mathrm{m}}^{l}\right)+\left(e\left(u^{l}\right), \nabla \psi^{l}\right)\right] d y,
\end{aligned}
$$

where $E^{k}=\left(E_{s}^{k}, E_{\mathrm{e}}^{k}, E_{\mathrm{m}}^{k}\right), k=1,2, \ldots, 12$, is any element of a basis $\mathbb{S}^{3} \times \mathbb{R}^{3} \times \mathbb{R}^{3}$ and $\left(u^{k}, \phi^{k}, \psi^{k}\right)$ are a solution to

$$
\left\{\begin{array}{l}
\text { Find }\left(u^{k}, \phi^{k}, \psi^{k}\right) \text { in } \mathcal{V}:=\left(H_{\#}^{1}\left(Y ; \mathbb{R}^{3}\right) / \mathbb{R}^{3}\right) \times\left(H_{\#}^{1}\left(Y_{\mathrm{e}}\right) / \mathbb{R}\right) \times\left(H_{\#}^{1}\left(Y_{\mathrm{m}}\right) / \mathbb{R}\right) ; \\
\int_{Y} \chi_{\mathrm{e}}(y) M_{\mathrm{e}}(y)\left(\left(E_{s}^{k}, E_{\mathrm{e}}^{k}\right)+\left(e\left(u^{k}\right), \nabla \phi^{k}\right)\right) \cdot(e(u), \nabla \phi) d y \\
\quad+\int_{Y} \chi_{\mathrm{m}}(y) M_{\mathrm{m}}(y)\left(\left(E_{s}^{k}, E_{\mathrm{m}}^{k}\right)+\left(e\left(u^{k}\right), \nabla \psi^{k}\right)\right) \cdot(e(u), \nabla \psi) d y \\
=L(u) \quad \forall(u, \phi, \psi) \in \mathcal{V} .
\end{array}\right.
$$


When $p=(1,2),(2,2),(2,4)$, the effective material is purely elastic, electricity and magnetism are only involved in the building of the effective elasticity tensor by solving cellproblems.

When $p=(1,3),(2,1),(2,3)$, the effective material is piezoelectric, magnetism is only involved in the building of the effective piezoelectric tensor.

When $p=(1,4)$, the effective material is piezomagnetic and electricity is only involved in the building of the effective piezomagnetic tensor.

\section{Discussion and conclusions}

From the mathematical point of view, the models obtained by $[2,3]$ were derived through formal homogenization approaches such as asymptotic expansions and field-averaging. The method presented here has the advantage of providing rigorous convergence results but also to enlightening the strategic aspects that must be taken into account by an engineer to design the proper multi-physical device with an effective electromagnetic coupling. Thus to have full piezoelectromagnetic behavior each phase has to be connected and the interface has to be impermeable. As the permeability/impermeability conditions are handily obtained through an additional coating of the phases of the composite, this opens the way for future investigations that will enrich our models.

Acknowledgements

Not applicable.

Funding

Not applicable.

Competing interests

The authors declare that they have no competing interests.

Authors' contributions

$\mathrm{CL}$ and TW conceived of the presented research work. $\mathrm{CL}$ and SO proved the main result, interpreted the result and wrote the paper. All authors read and approved the final manuscript.

Authors' information

Christian Licht email: christian.licht@umontpellier.fr; Thibaut Weller email: thibaut.weller@umontpellier.fr.

\section{Author details}

'Department of Mathematics, Faculty of Science, Mahidol University, Bangkok, Thailand. ${ }^{2}$ LMGC-UMR 5508, Université de Montpellier-CC048, Montpellier, France. ${ }^{3}$ Centre of Excellence in Mathematics, CHE, Bangkok, Thailand.

\section{Publisher's Note}

Springer Nature remains neutral with regard to jurisdictional claims in published maps and institutional affiliations.

Received: 29 January 2019 Accepted: 2 May 2019 Published online: 14 May 2019

\section{References}

1. Van Suchtelen, J.: Product properties: a new application of composite materials. Philips Res. Rep. 27, 28-37 (1972)

2. Nguyen, T.T.: Etude des comportements statique et dynamique des composites à phases piézo-électrique et piézo-magnétique. Thesis, Université Paris XIII, Sorbonne Paris Cité, Institut Galillée, Laboratoire des Sciences des Procédés et des Matériaux (2016)

3. Nguyen, T.T., Abdelmoula, R., Li, J., Roussigne, Y., Stashkevich, A.: Wave propagating in multilayers composed of piezoelectric and piezomagnetic layers. Composites, Part B, Eng. 93, 289-301 (2016)

4. Allaire, G.: Homogenization and two-scale convergence. J. Math. Anal. 23, 1482-1518 (1992)

5. Allaire, G., Murat, F.: Homogenization of the Neumann problem with nonisolated holes. Asymptot. Anal. 7, 81-95 (1993) 